\title{
Am I Floating or Not? : Sensitivity to Eye Height Manipulations in HMD-based Immersive Virtual Environments
}

\author{
Zhihang Deng \\ Victoria Interrante \\ Department of Computer Science, University of Minnesota
}

\begin{abstract}
Eye height manipulations have previously been found to affect judgments of object size and egocentric distance in both real and immersive virtual environments. In this short paper we report the results of an experiment that explores people's sensitivity to various offsets of their eye height in VR using a forced-choice task in a wide variety of different architectural models. Our goal is to better understand the range of eye height manipulations that can be surreptitiously employed under different environmental conditions.

We exposed each of 10 standing participants to a total of 121 randomly-ordered trials, spanning 11 different eye height offsets between $-80 \mathrm{~cm}$ to $+80 \mathrm{~cm}$, in 11 different highly detailed virtual indoor environments, and asked them to report whether they felt that their (invisible) feet were floating above or sunken below the virtual floor. We fit psychometric functions to the pooled data and to the data from each virtual environment and each participant individually. In the pooled data, we found a point-of-subjective-equality (PSE) very close to zero $(-3.8 \mathrm{~cm})$, and $25 \%$ and $75 \%$ detection thresholds of $-16.1 \mathrm{~cm}$ and $+8.6 \mathrm{~cm}$ respectively, for an uncertainty interval of $24.7 \mathrm{~cm}$. We also observed some interesting variations in the results between individual rooms, which we discuss in more detail in the paper. Our findings can help to inform VR developers about users' sensitivity to incorrect eye height placement, to elucidate the potential impact of various features of interior spaces on people's tolerance of eye height manipulations, and to inform future work seeking to employ eye height manipulations to mitigate distance underestimation in VR.
\end{abstract}

\section{CCS CONCEPTS}

- Human-centered computing $\rightarrow$ Virtual reality; • Computing methodologies $\rightarrow$ Perception.

\section{KEYWORDS}

Virtual reality, spatial perception, eye height

\section{ACM Reference Format:}

Zhihang Deng and Victoria Interrante. 2019. Am I Floating or Not? : Sensitivity to Eye Height Manipulations in HMD-based Immersive Virtual Environments. In ACM Symposium on Applied Perception 2019 (SAP '19),

Permission to make digital or hard copies of all or part of this work for personal or classroom use is granted without fee provided that copies are not made or distributed for profit or commercial advantage and that copies bear this notice and the full citation on the first page. Copyrights for components of this work owned by others than ACM must be honored. Abstracting with credit is permitted. To copy otherwise, or republish, to post on servers or to redistribute to lists, requires prior specific permission and/or a fee. Request permissions from permissions@acm.org.

SAP '19, September 19-20, 2019, Barcelona, Spain

(C) 2019 Association for Computing Machinery.

ACM ISBN 978-1-4503-6890-2/19/09..\$15.00

https://doi.org/10.1145/3343036.3343135
September 19-20, 2019, Barcelona, Spain. ACM, New York, NY, USA, 6 pages. https://doi.org/10.1145/3343036.3343135

\section{INTRODUCTION}

In most immersive virtual reality (VR) experiences, the user inherently interprets what they see as if it were a view from their own eyes [Leyrer et al. 2015b]. Several common and uncommon situations have the potential to create a dissociation between the user's simulated eye height in VR and their concurrent actual eye height in the real world, however. When multiple users all share the same view, for instance on an immersive projection display, only one of them will see the virtual environment from their own eye height, and this situation has been associated with errors in spatial judgments about the depicted scene [Pollock et al. 2012]. VR applications designed for seated use may not adjust the presented viewpoint to match the user's actual eye height, either from a standing or seated vantage point. Some applications provide the user with locomotion controls that allow unconstrained 3DOF movement in VR; the relationship between the virtual viewpoint and the user's real world eye height can then be rapidly lost. Finally, some applications deliberately manipulate the user's eye height in VR to achieve various purposes, such as to support the illusion of being a giant [Kim and Interrante 2017] or to help users make more accurate judgments of egocentric distances [Leyrer et al. 2011].

Our goal in this paper is to seek a better understanding of people's sensitivity to discrepancies between their actual standing eye height in the real world and the height off the floor of their virtual vantage point in VR. This information has the potential to inform developers of VR applications how much leeway they are likely to have in pre-setting or adjusting a user's eye height in VR before they would be likely to notice that their eye height is wrong. For applications that rely on eye height manipulations, it is possible that surreptitious manipulations could have different effects than manipulations that are overt.

\section{RELATED WORK}

Egocentric distance perception has been well-studied in both VR and the real world. A large body of prior work focuses on egocentric judgments of horizontal distances over a groundplane, which are generally found to be underestimated in VR relative to in the real world [Renner et al. 2013]. A much smaller body of work has specifically looked at egocentric distance perception in the vertical direction; it is reported to be generally overestimated, especially from the top looking down [Stefanucci and Proffitt 2009]. In VR, most research has found that embodiment in a size-matched selfavatar enables more accurate judgments of egocentric distances both horizontally [Mohler et al. 2010; Ries et al. 2008] and vertically [Lin et al. 2015]. 
There is a modest body of research on the impact of eye height offsets on size and distance perception. Wraga [1999] used a false floor in real world experiments to surreptitiously manipulate people's effective eye height in relation to the groundplane on which objects could be seen to sit and found a strong effect of the eye height manipulation on object size (height) perception, for both seated and standing observers. Dixon et al. [2000] found that surreptitious eye height manipulations in VR had a similar effect on size perception for generic objects resting on the same groundplane as the observer. Kim and Interrante [2017], however, found no impact of overt eye height manipulations on users' perception of the size of a generic object embedded in a richly detailed realistic virtual room.

Langbehn et al. [2016] investigated participants' sense of whether it was they or their environment that had changed size after coordinated manipulations of eye height and IPD and found that participants were more likely to believe that their surrounding environment, rather than they themselves, had changed size when they were embodied than when not. Piumsomboon et al. [2018] found that stereo disparity (IPD) in conjunction with eye height

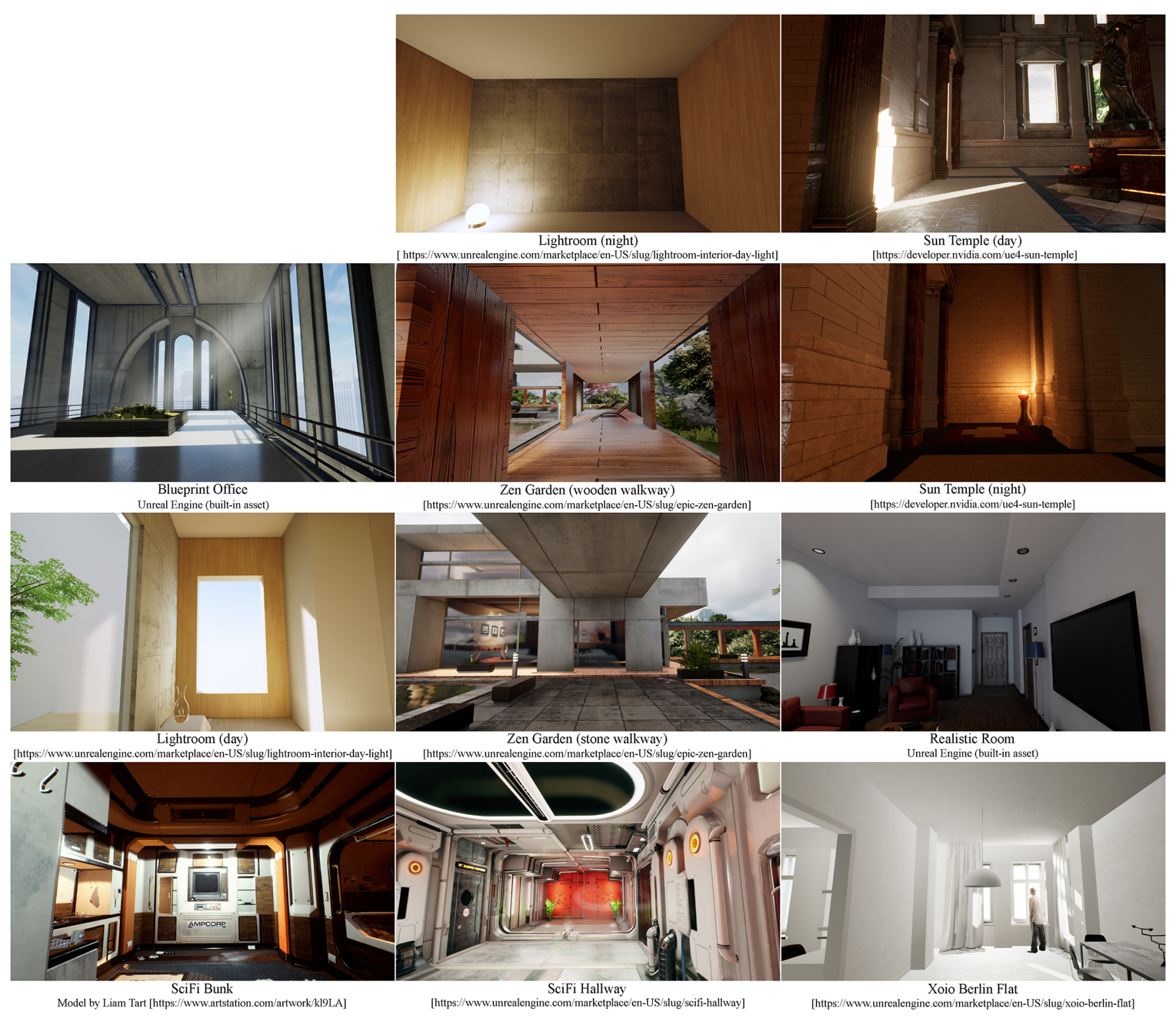

Figure 1: Views of each of the virtual environments used in our experiment, taken from an eye height of $150 \mathrm{~cm}$ and facing in the default initial direction. The Lightroom model was created by "Koola". The Berlin Flat model was created by Xoio. The SciFi Bunk model was created by Liam Tart. The Sun Temple [Games 2017], Zen Garden, Scifi Hallway, Realistic Room and Blueprint Office models were created by Epic Games. 


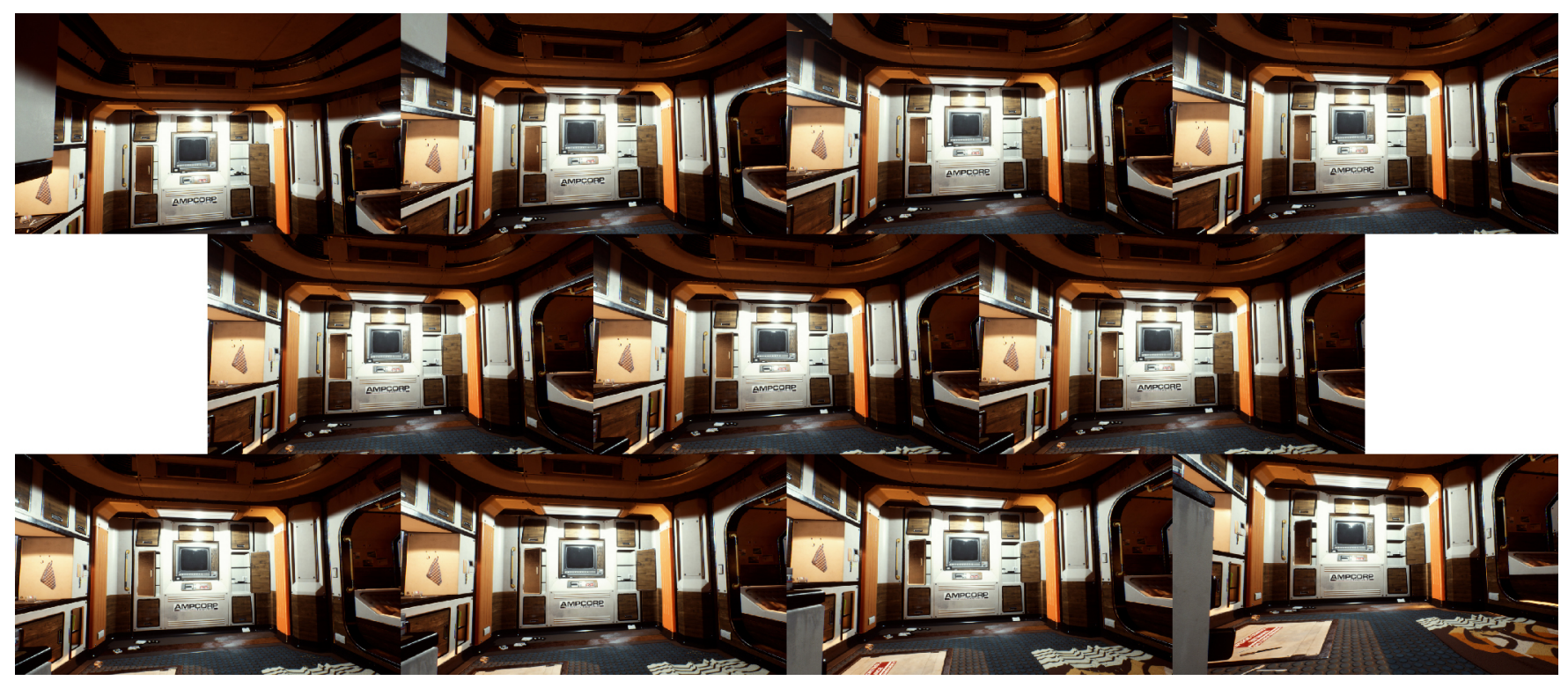

Figure 2: Views of the SciFi Bunk with each of the different eyeheight offsets applied. Top row (left to right): $+80 \mathrm{~cm},+32 \mathrm{~cm}$, $+12 \mathrm{~cm},+5 \mathrm{~cm}$; Middle row: $+2 \mathrm{~cm}, 0 \mathrm{~cm},-2 \mathrm{~cm}$; Bottom row: $-5 \mathrm{~cm},-12 \mathrm{~cm},-32 \mathrm{~cm},-80 \mathrm{~cm}$.

manipulations influenced people's sense of being a giant versus flying in VR when no embodiment was visible.

Leyrer et al. [2011] found that eye height manipulations of $+50 \mathrm{~cm}$ or $-50 \mathrm{~cm}$ had a significant impact on participants' judgments of egocentric distance in a highly realistic indoor environment and were unnoticed by participants when a between-subjects experimental design was used; Leyrer et al. [2015a] showed that eye height manipulations could be successfully used to correct for distance estimation errors in VR in an individualized way, regardless of whether the eye height manipulation was noticed or not.

\section{OUR EXPERIMENT}

As eye height offsets have been shown to affect judgments of size and distance in VR, and situations arise in which such offsets may occur (either by design or by accident), we felt that it would be useful to investigate the extent to which and conditions under which people are likely to be aware of the existence of a displacement between their standing eye height and the altitude of their virtual viewpoint.

\subsection{Design}

We used a within-subjects, forced-choice experiment in which participants were asked to report the direction ("above" or "below") of the offset of their virtual viewpoint over 121 total trials spanning 11 different offsets ranging from $-80 \mathrm{~cm}$ to $+80 \mathrm{~cm}$ in approximately equal $\log$ intervals $(-80,-32,-12,-5,-2,0,+2,+5,+12,+32,+80)$ in each of 11 different richly detailed architectural environments containing both floor and ceiling surfaces. Figure 1 shows what each of these environments looks like. Figure 2 shows what each of the different eyeheight offsets looks like, applied in the SciFi Bunk model.

\subsection{Participants}

We recruited 10 participants $(6 \mathrm{M}, 4 \mathrm{~F})$, ages $21-34(\mu=25.9 \pm 4.7)$, by word of mouth from our local university community. Participants were compensated with a $\$ 10$ gift card to an online retailer.

\subsection{Materials}

Participants viewed the virtual environment using an HTC Vive head-mounted display, which weighs $\sim 1 \mathrm{lb}$ and has two $1080 \times 1200$ OLED displays, one for each eye, presenting a stereo view over a combined $100^{\circ} \mathrm{h} \times 110^{\circ} \mathrm{v}$. We used Valve's Lighthouse tracking system to dynamically track the position and orientation of the HMD within a $\sim 15^{\prime} \times \sim 15^{\prime}$ area at one end of a larger open lab space; the eye height offset was added to this tracked position as a constant displacement in the $\mathrm{z}$-direction. The virtual environment models were all obtained as free assets from the Unreal Engine Marketplace or are built-in assets in the Unreal game engine. The virtual environment was rendered using Unreal Engine on an ORIGIN PC with an Intel Core i7 6850K Hex-Core $3.6 \mathrm{GHz}$ processor, 32GB DDR4 SDRAM $(2800 \mathrm{MHz})$, and a single 8GB NVIDIA GeForce GTX 1080 Founders Edition graphics card.

\subsection{Procedure}

Participants were screened for normal visual acuity and stereo vision ability. After giving written informed consent, they were asked to read written instructions explaining the experiment procedure. The instructions informed them that they would be immersed in a series of different virtual interior and exterior spaces, and in each one they should briefly look around (without walking to a different position) and then verbally indicate whether they felt that their feet (which we told them they would not be able to see in VR) would be floating above ("above") or sunken below ("below") the virtual floor, if they were not invisible. 
At the start of the experiment, the participant stood at a comfortable location within the tracked space and put on the HMD and a pair of noise-cancelling headphones. The experimenter verified that the headset was properly secured. Each participant completed a total of 121 trials in each of which they were immersed in one of a series of 11 different virtual environments with one of 11 different eye height offsets. The 121 stimuli were presented in a different random order for each participant. To ensure comfortable transitions and discourage pairwise comparisons of eye height between trials, the participant's view was faded out to black after they made each judgment, and remained black for a few seconds before the next environment faded in. To avoid fatigue, participants were invited to take a short break after 44 and 88 trials, during which time they were invited to enjoy some sparkling water and snacks. Participants were allowed to proceed at their own pace and everyone completed all of the trials in under 1 hour (most took only about 30 minutes).

\section{RESULTS}

We used the psignifit toolbox ${ }^{1}$ to fit a psychometric function to the pooled data from all 10 participants (Figure 3), with a goodnessof-fit of 0.00062 . We found a very slight overestimation of eye height, corresponding to a PSE of $-3.8 \mathrm{~cm}$, and a modest uncertainty interval of $24.7 \mathrm{~cm}$, with $25 \%$ of participants responding "above" at an eyeheight offset of $-16.1 \mathrm{~cm}$ and $75 \%$ responding "above" at an eyeheight offset of $8.6 \mathrm{~cm}$. The horizontal error bars around the PSE and each of the endpoints of the uncertainty interval indicate the $95 \%$ confidence intervals for each of these values, provided by psignifit 4 [Schütt et al. 2016].

We also fit psychometric functions to the data from each room separately (Figure 4). Due to the smaller number of data points, the average goodness-of-fit for these curves was only 0.0079 (about $10 \mathrm{x}$ worse than for the pooled data). We found a modest variation of the uncertainty intervals between the different rooms, as well

\footnotetext{
${ }^{1}$ https://github.com/wichmann-lab/psignifit/archive/master.zip
}

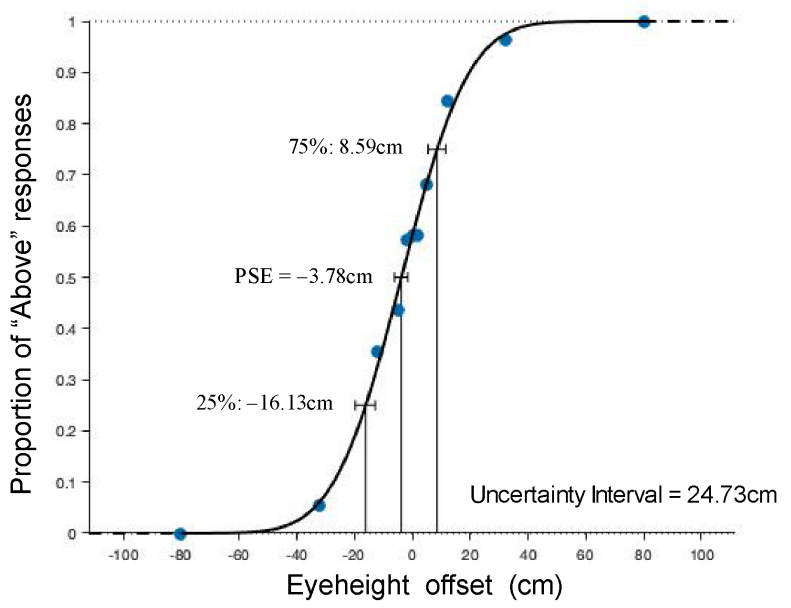

Figure 3: A psychometric fit to the pooled data from all trials by all participants.
Table 1: Individual Participants’ Data

\begin{tabular}{ccccc}
\hline EyeHeight & $25 \%$ & PSE & $75 \%$ & Uncertainty Interval \\
\hline $152 \mathrm{~cm}$ & $78.68 \%$ & $79.23 \%$ & $79.79 \%$ & $1.10 \%$ \\
$142 \mathrm{~cm}$ & $77.71 \%$ & $84.99 \%$ & $92.26 \%$ & $14.56 \%$ \\
$161 \mathrm{~cm}$ & $89.34 \%$ & $96.09 \%$ & $102.84 \%$ & $13.49 \%$ \\
$159 \mathrm{~cm}$ & $92.32 \%$ & $97.83 \%$ & $103.33 \%$ & $11.01 \%$ \\
$171 \mathrm{~cm}$ & $91.38 \%$ & $98.88 \%$ & $106.38 \%$ & $15.00 \%$ \\
$140 \mathrm{~cm}$ & $90.63 \%$ & $99.14 \%$ & $107.66 \%$ & $17.04 \%$ \\
$161 \mathrm{~cm}$ & $97.75 \%$ & $100.78 \%$ & $103.81 \%$ & $6.05 \%$ \\
$145 \mathrm{~cm}$ & $98.68 \%$ & $102.73 \%$ & $106.78 \%$ & $8.09 \%$ \\
$157 \mathrm{~cm}$ & $96.89 \%$ & $103.51 \%$ & $110.12 \%$ & $13.23 \%$ \\
$142 \mathrm{~cm}$ & $96.73 \%$ & $105.02 \%$ & $113.31 \%$ & $16.58 \%$ \\
\hline
\end{tabular}

as some interesting differences in the PSE. Details are provided in Figure 4 and discussed in section 5 below.

Finally, we additionally fit separate psychometric functions to the data from each participant (Table 1), with each offset represented as a percentage of the participant's eye height. Eight of the ten participants had a PSE within $5 \%$ of their actual eye height, but the other two exhibited a strong bias to respond "above".

\section{DISCUSSION}

While our general finding of a slight overestimation of eye height is consistent with the findings of Stefanucci and Proffitt [2009], it may also be somewhat skewed by the responses of the 1-2 participants who more rarely reported the impression of their feet being under the virtual floor, despite the fact that the confidence interval around the PSE is entirely within the negative range. Replicating the experiment with a larger number of participants, or re-doing it using a potentially more sensitive psychometric method, would allow this observation to be stated with greater certainty. Overall, we note that the majority of our participants seemed relatively sensitive to modest eye height manipulations when they knew to look for them, a finding that expands upon the observations of Leyrer et al. [2015a] whose participants typically did not report awareness of eye height manipulations up to $\pm 50 \mathrm{~cm}$ when the manipulations were encountered in a between-subjects design.

We also note that uncertainty intervals for eye height manipulations tended to be smallest in the scenes that contained elements capable of providing strong familiar size cues, such as people, doors, and items of furniture that are ergonomically-constrained in their design. Previous research has shown that familiar objects not only have an assumed canonical size, which can influence the perception of their distance [Epstein 1963] but also that people tend to recall familiar objects from a preferred canonical perspective that reflects, in part, their accumulated visual experiences with them [Konkle and Oliva 2011]. This might explain why uncertainty intervals tended to be larger in the scenes that featured larger-than-typical spaces and higher-than-typical ceilings, such as the Blueprint Office and Sun Temple, or that lacked familiar features that could serve as reliable points of reference for judging eye height, such as the Lightroom environment in the nighttime viewing configuration. However, we caution that due to the small number of participants, the amount of 


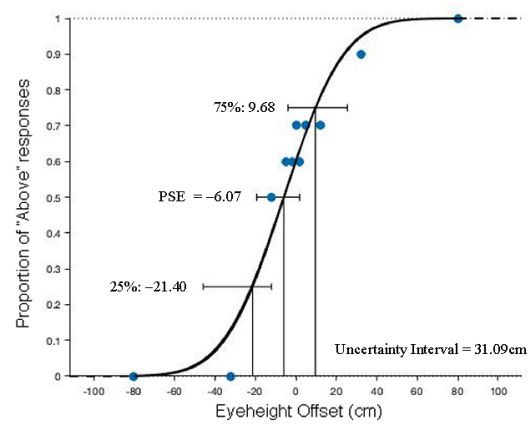

Lightroom (night)

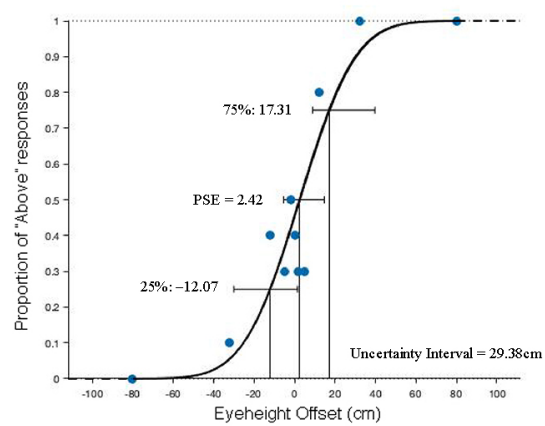

Blueprint Office

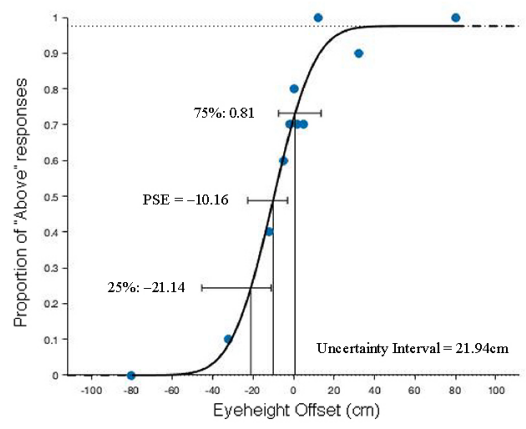

Lightroom (day)

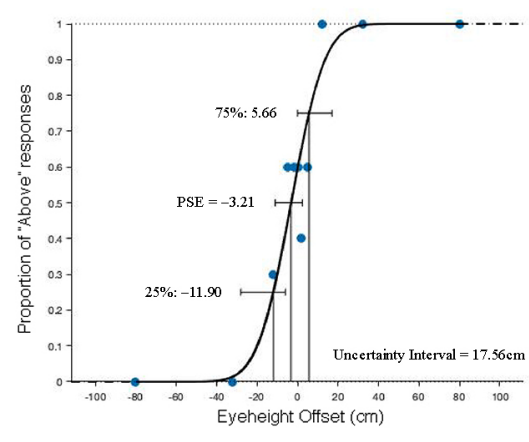

SciFi Bunk

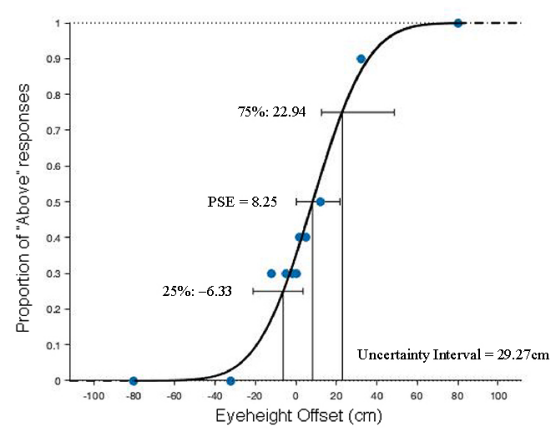

Zen Garden (wooden walkway)

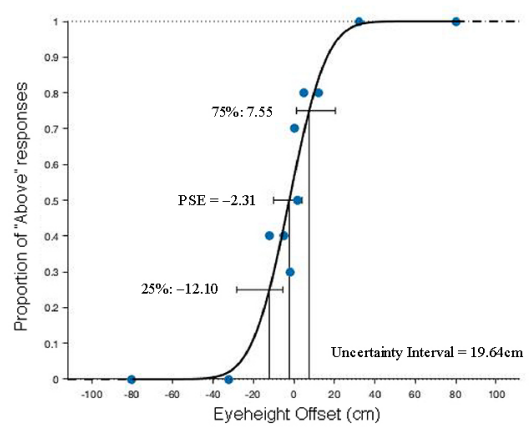

Zen Garden (stone walkway)

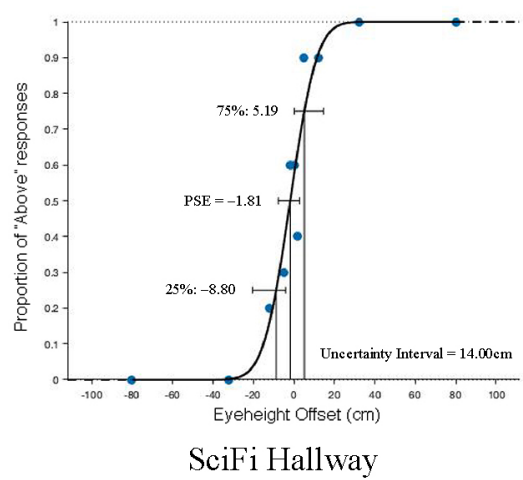

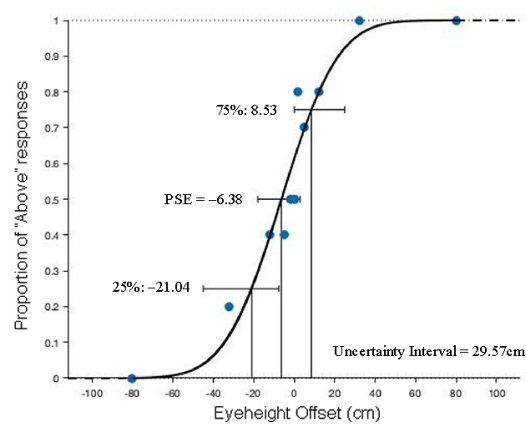

Sun Temple (day)

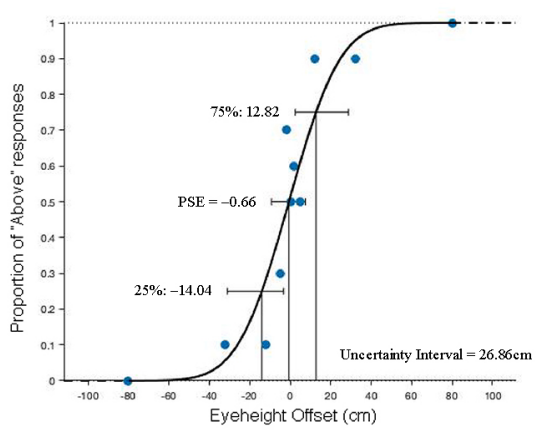

Sun Temple (night)

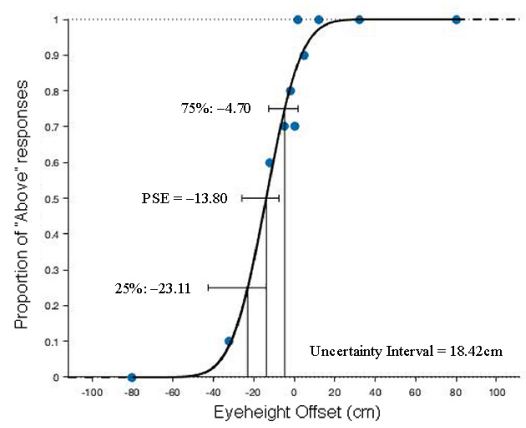

Realistic Room

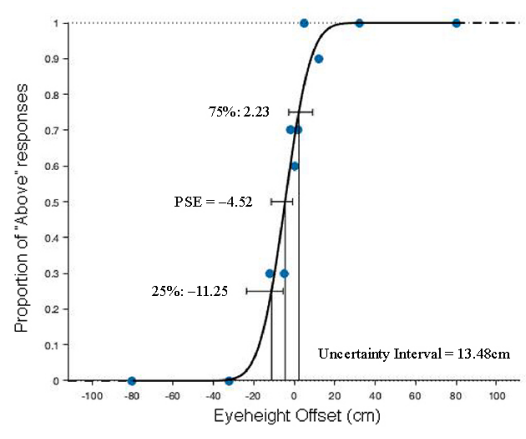

Xoio Berlin Flat

Figure 4: Plots of the psychometric fits to the data for each room separately. 
data available for each room is likely insufficient to enable robust pairwise comparisons.

\section{CONCLUSIONS AND FUTURE WORK}

Our small study makes a modest first step towards a better understanding of the range of offsets over which eye height manipulations are likely to go unnoticed by standing users in common VR scenarios. In future work, we plan to extend these findings through additional experiments using more sensitive psychometric methods, with more participants, considering a seated as well as standing posture, and incorporating a self-avatar. It also remains an interesting open question the extent to which people might be able to use the awareness of the existence of an offset in their eye height to compensate for the perceptual distortions that would otherwise be expected to occur [Vishwanath et al. 2005]. The answers to that question could be informative to video-see-through applications that rely on cameras that are offset from the eyes' positions.

\section{ACKNOWLEDGMENTS}

This research was supported by the National Science Foundation (CHS 1526693) and by the Linda and Ted Johnson Digital Design Consortium Endowment. We would like to thank the anonymous reviewers for their very helpful suggestions about how to improve and extend this work.

\section{REFERENCES}

Melissa W. Dixon, Maryjane Wraga, Dennis R. Proffitt, and George C. Williams. 2000 Eye Height Scaling of Absolute Size in Immersive and Nonimmersive Displays. Fournal of Experimental Psychology: Human Perception and Performance 26, 2 (2000), 582-593. https://doi.org/10.1037/0096-1523.26.2.582

William Epstein. 1963. The influence of assumed size on apparent distance. American Journal of Psychology 76 (1963), 257-265. Issue 2. https://doi.org/10.2307/1419162

Epic Games. 2017. Unreal Engine Sun Temple, Open Research Content Archive (ORCA) http://developer.nvidia.com/orca/epic-games-sun-temple

Jangyoon Kim and Victoria Interrante. 2017. Dwarf or Giant: The Influence of Interpupillary Distance and Eye Height on Size Perception in Virtual Environments. In Proceedings of the International Conference on Artificial Reality and Telexistence and Eurographics Symposium on Virtual Environments (ICAT-EGVE '17). Eurographics Association, Goslar Germany, Germany, 153-160. https://doi.org/10.2312/egve 20171353

Talia Konkle and Aude Oliva. 2011. Canonical visual size for real-world objects. fournal of Experimental Psychology: Human Perception and Performance 37 (2011), 23-37. Issue 1. https://doi.org/10.1037/a0020413

Eike Langbehn, Gerd Bruder, and Frank Steinicke. 2016. Scale Matters ! Analysis of Dominant Scale Estimation in the Presence of Conflicting Cues in Multi-Scale Collaborative Virtual Environments. In Proceedings of the IEEE Symposium on $3 D$ User Interfaces (3DUI). IEEE, Piscataway, NJ, USA, 211-220. https://doi.org/10.1109/ 3DUI.2016.7460054

Markus Leyrer, Sally A. Linkenauger, Heinrich H. Bülthoff, Uwe Kloos, and Betty Mohler. 2011. The Influence of Eye Height and Avatars on Egocentric Distance Estimates in Immersive Virtual Environments. In Proceedings of the ACM Symposium on Applied Perception in Graphics and Visualization (APGV '11). ACM, New York, NY, USA, 67-74. https://doi.org/10.1145/2077451.2077464

Markus Leyrer, Sally A. Linkenauger, Heinrich H. Bülthoff, and Betty J. Mohler. 2015a Eye Height Manipulations: A Possible Solution to Reduce Underestimation of Egocentric Distances in Head-Mounted Displays. ACM Transactions on Applied Perception 12, 1, Article 1 (Feb. 2015), 23 pages. https://doi.org/10.1145/2699254

Markus Leyrer, Sally A. Linkenauger, Heinrich H. Bülthoff, and Betty J. Mohler. 2015b. The Importance of Postural Cues for Determining Eye Height in Immersive Virtual Reality. PLoS ONE 10, 5 (2015), 1-23. https://doi.org/10.1371/journal.pone.0127000

Qiufeng Lin, John Rieser, and Bobby Bodenheimer. 2015. Affordance Judgments in HMD-Based Virtual Environments : Stepping Over a Pole and Stepping Off a Ledge. ACM Transactions on Applied Perception 12, 2 (2015), 1-21. https://doi.org/10.1145/ 2720020

Betty J. Mohler, Sarah H. Creem-Regehr, and William B. Thompson. 2010. The Effect of Viewing a Self-Avatar on Distance Judgments in an HMD-Based Virtual Environment. Presence: Teleoperators and Virtual Environments 19, 3 (2010), 230-242. https://doi.org/10.1162/pres.19.3.230

Thammathip Piumsomboon, Gun A. Lee, Barrett Ens, Bruce H. Thomas, and Mark Billinghurst. 2018. Superman vs Giant : A Study on Spatial Perception for a MultiScale Mixed Reality Flying Telepresence Interface. IEEE Transactions on Visualization and Computer Graphics 24, 11 (2018), 2974-2982. https://doi.org/10.1109/ TVCG.2018.2868594

Brice Pollock, Melissa Burton, Jonathan W. Kelly, Stephen Gilbert, and Eliot Winer. 2012. The Right View from the Wrong Location : Depth Perception in Stereoscopic Multi-User Virtual Environments. IEEE Transactions on Visualization and Computer Graphics 18, 4 (2012), 581-588. https://doi.org/10.1109/TVCG.2012.58

Rebekka S. Renner, Boris M. Velichkovsky, and Jens R. Helmert. 2013. The Perception of Egocentric Distances in Virtual Environments - A Review. Comput. Surveys 46, 2 (2013), 1-40. https://doi.org/10.1145/2543581.2543590 arXiv:arXiv:1502.07526v1

Brian Ries, Victoria Interrante, Michael Kaeding, and Lee Anderson. 2008. The Effect of Self-Embodiment on Distance Perception in Immersive Virtual Environments. In Proceedings of the ACM Symposium on Virtual Reality Software and Technology (VRST '08). ACM, New York, NY, USA, 167-170. https://doi.org/10.1145/1450579.1450614

Heiko H. Schütt, Stefan Harmeling, Jakob H. Mackee, and Felix A. Wichmann. 2016. Painfree and Accurate Bayesian Estimation of Psychometric Functions for (Potentially) Overdispersed Data. Vision Research 122 (2016), 105-123. https: //doi.org/10.1016/j.visres.2016.02.002

Jeanine K Stefanucci and Dennis R Proffitt. 2009. The Roles of Altitude and Fear in the Perception of Height. Fournal of Experimental Psychology: Human Perception and Performance 35, 2 (2009), 424-438. https://doi.org/10.1037/a0013894

Dhanraj Vishwanath, Ahna R. Girshick, and Martin S. Banks. 2005. Why Pictures Look Right When Viewed From the Wrong Place. Nature Neuroscience 8 (2005), 1401-1410. Issue 10. https://doi.org/10.1038/nn1553

Maryjane Wraga. 1999. Using Eye Height in Different Postures to Scale the Heights of Objects. Fournal of Experimental Psychology: Human Perception and Performance 25, 2 (1999), 518-530. https://doi.org/10.1037/0096-1523.25.2.518 